\title{
Formation of Elements and Compounds in Space in Early Universe
}

\author{
Abdul L. Bhuiyan ${ }^{1}$ \\ ${ }^{1}$ Box 13, Head Post office, Comilla-3500, Bangladesh \\ Correspondence: Abdul L. Bhuiyan, Box 13, Head Post office, Comilla-3500, Bangladesh. E-mail: \\ ananda.computers@yahoo.com
}

Received: November 1, 2016

Accepted: November 18, 2016

Online Published: November 30, 2016

doi:10.5539/apr.v8n6p86

URL: http://dx.doi.org/10.5539/apr.v8n6p86

\begin{abstract}
At the end of the period of contraction of the universe, all objects transform into gravity particles such as photons and electron- positron pairs which exist in virtual state in spacetime at an extremely high temperature. These particles move with extremely high speed comparable to the speed of light. As the early universe starts cooling, the speed of the particles starts to decrease when photons and electron- positron pairs move out of spacetime and appear as real particles. As the temperature continues to fall due to cooling, the electron- positron pairs start forming quarks ( $u$ and $d$ ) while simultaneously the energy of photons transform into dark matter. The $u$ quarks and $\mathrm{d}$ quarks then continue to form nuclei of different elements including radio elements. Simultaneously, the lighter elements such as hydrogen, nitrogen, carbon, oxygen, phosphorus, etc. form the precursors to DNAs and RNAs of living organisms.
\end{abstract}

Keywords: Pathways of formation of nuclei, Nuclear polymers, Nuclear fission, Precursors of living organisms in space, natural modification of genes

\section{Introduction}

The singularity theorems deduced by using the theory of general relativity show that time (as well as the spacetime universe) starts from a Big Bang singularity which is a point of infinite density and infinite curvature of spacetime (Hawking, 1988). Also, attempts to reconcile the general relativity with the principles of quantum mechanics runs into the renormalization process. It was soon found that the sum of all the forces in this process does not become zero and consequently the result is infinite value. But all these infinite values cannot be used to make meaningful physical predictions, for which the theory of general relativity cannot be used to predict the events which could lead to the occurrence of Big Bang if the concept of Big Bang singularity is correct. For this, it is needed to use some other ways to find out the processes that occurred at the beginning of the universe.

In a previous paper (Bhuiyan, 2010), it was deduced by using quantum mechanics and uncertainty principle that the universe performs simple harmonic motion between expansion and contraction and that at the end of the period of contraction : (a) the universe assumes the smallest possible finite size with no boundary, (b) all the objects are transformed into quantized gravity / gravitational force of attraction called gravity particles which move with speed comparable to the speed of light, (c) the density and temperature of the gravity particles become extremely high, and (d) the gravitational force of attraction between the gravity particles becomes extremely strong. As the universe is of finite size and has no boundary, there does not exist any point of Big Bang singularity; that is, the Big Bang is now cut out of the theory of universe.

According to Sekine (Sekine, 2012), quarks appeared in the first moments after the Big Bang. As the Big Bang singularity does not exist, one can conclude from this statement that quarks appeared perhaps sometimes at the beginning of the expansion of the universe. As soon as the quarks had appeared, they started to cluster in pairs. These clusters then started to form atomic nuclei (Bhuiyan, 2013; Bhuiyan, 2016). But u quarks and d quarks are composed of electron- positron pairs (Bhuiyan, 2016), suggesting that electron- positron pairs had appeared before the appearance of quarks. Since photons are annihilation products of electron- positron pairs, both photons and electron- Positron pairs appeared simultaneously, showing that all objects were transformed into photons in reversible equilibrium with electron- positron pairs at the end of the period of contraction, or at the beginning of the period of expansion, of the universe. The force of gravity carried by a photon is given by (Bhuiyan, 2015).

$$
F=\frac{n h t c^{2}}{2 a^{3}}
$$


or,

$$
F=\frac{n h c^{2}}{2 a \times a \times a \times \frac{1}{t}}
$$

where the quantity $a \times a \times a \times \frac{1}{t}$ may represent the volume of four-dimensional cell (or box), whose three sides are made of space and the fourth side is made of reciprocal of time. The presence of $n$ indicates that space and time are quantized and that spacetime cells are made of these quanta. The presence of $\mathrm{n}$ also indicates that the mass, energy and force carried by photons are space-time quantized. In a nutshell, the space-time quantized photons move in the virtual state in spacetime with the speed of light at the end of the period of contraction (or just at the beginning of the period of expansion). Since photons carry quantized gravity/gravitation, they may be comparable to the gravity particles (Bhuiyan, 2010). Thus, the Big Bang singularity is now replaced by the embryo universe of space- time quantized photons, governed by quantum mechanics and uncertainty principle, which existed in reversible equilibrium with electron - positron pairs.

As the embryo universe starts to cool, the kinetic energy of the photons and the electron- positron pairs in virtual state in spacetime starts to decrease, which in turn causes the speed of these particles to decrease.

As a result, these particles move out of the virtual state and behave as real particles. On further cooling, the photons start loosing more kinetic energy to dark matter and simultaneously the electron- positron pairs start forming $\mathrm{u}$ quarks and d quarks (Bhuiyan, 2016). Starting with u quarks and d quarks, the chains of formation of different nuclei are now described in the following sections. However, the formation of these chains might depend upon the relative proportions of $u$ quarks and d quarks in different regions of space in the early universe.

\section{Number of u Quarks Equal to that of d Quarks.}

Due to high density of the particles in the early universe, a u quark might undergo inelastic collision with a d quark to produce a ud pair; thus

$$
\mathrm{u} \quad+\mathrm{d} \rightarrow \mathrm{ud}
$$

The two quarks in the ud pair are bonded to each other through a double bond (Bhuiyan, 2016), for which these pairs might polymerize to form the polymers (-u-d-) $)_{x}$ of nuclei as follows. The charge on a ud pair is $+1 / 3$. So, the total charge on three ud pairs is $3 X(+1 / 3)=+1$. This indicates that a set of three ud pairs would produce the smallest nucleus of charge equal to +1 , suggesting that $\mathrm{x}$ in the polymers $(-\mathrm{u}-\mathrm{d}-)_{\mathrm{x}}$ should be substituted with $3 \mathrm{n}$, where $\mathrm{n}=$ $1,2,3$, etc., so that the polymer $(-\mathrm{u}-\mathrm{d}-)_{\mathrm{x}}$ assumes the form $(-\mathrm{u}-\mathrm{d}-)_{3 n}$. For each value of $\mathrm{n}$, the polymer $(-\mathrm{u}-\mathrm{d}-)_{3 \mathrm{n}}$ produces a nucleus of clusters of ud pairs. For smallest value of $n(n=1)$, the polymer $(-u-d-)_{3 n}$ produces $3 u d$. But 3 ud represents the smallest compound nucleus of (uud+udd) cluster which possesses charge equal to +1 .

\section{More u Quarks than d Quarks.}

As d quarks are smaller in numbers, all of them may unite with the same number of $u$ quarks to produce ud pairs. Each of the remaining u quarks may emit a positron and transform into a d quark. Immediately after formation, the $\mathrm{d}$ quark may form a ud pair with an unconverted $\mathrm{u}$ quark. This process continues till all the unpaired $\mathrm{u}$ quarks are converted into ud pairs. For example, let there be present twenty-eight unpaired u quarks and fourteen unpaired $\mathrm{d}$ quarks in a region of space. The chain of formation of probable nuclei may be described as follows.

$$
\begin{gathered}
(28 \mathrm{u}+14 \mathrm{~d}) \rightarrow(14 \mathrm{ud}+14 \mathrm{u}) \\
\stackrel{e^{+}}{\longrightarrow}(15 \mathrm{ud}+12 \mathrm{u}, \mathrm{Al}-14(13 \mathrm{uud}+\mathrm{udd}) \\
\stackrel{e^{+}}{\longrightarrow}(16 \mathrm{ud}+10 \mathrm{u}, \mathrm{Mg}-14(12 \mathrm{uud}+2 \mathrm{udd}) \\
\stackrel{e^{+}}{\longrightarrow}(17 \mathrm{ud}+8 \mathrm{u}, \mathrm{Na}-14(11 \mathrm{uud}+3 \mathrm{udd}) \\
\stackrel{e^{+}}{\longrightarrow}(18 \mathrm{ud}+6 \mathrm{u}, \mathrm{Ne}-14(10 \mathrm{uud}+4 \mathrm{udd}) \\
\stackrel{e^{+}}{\longrightarrow} \quad(19 \mathrm{ud}+4 \mathrm{u}, \mathrm{F}-14(9 \mathrm{uud}+5 \mathrm{udd})
\end{gathered}
$$




$$
\begin{gathered}
\stackrel{e^{+}}{\longrightarrow} \quad(20 \mathrm{ud}+2 \mathrm{u}, 0-14(8 \mathrm{uud}+6 \mathrm{udd}) \\
\stackrel{e^{+}}{\longrightarrow} \text { (21ud, N-14 (7uud+7udd) }
\end{gathered}
$$

Thus, the nuclei Al-14 through N-14 are isobars of which the N-14 nucleus is most stable. It is noteworthy that with more $u$ quarks than d quarks, the chain of transformation describes the formation of nuclei of lower atomic numbers from unstable nuclei of higher atomic numbers. So, the nuclei containing unpaired u quarks tend to emit positrons and transform into nuclei of lower atomic numbers. This may be attributed to the instability of the unpaired $\mathrm{u}$ quark due to repulsive interaction between it and the positively charged nuclear ud pairs. As N-14 nucleus may also be obtained from the polymer ( $-\mathrm{u}-\mathrm{d}-)_{3 n}$ for $n=7$, and since $\mathrm{N}-14$ contains no unpaired quarks, the nuclei represented by (-u-d-) $3 n$ should also be stable like N-14 nucleus.

\section{More d Quarks than u Quarks.}

Since u quarks are smaller in numbers, all of them may unite with the same number of d quarks to produce ud pairs. Each of the remaining d quarks may emit an electron and transform into a $u$ quark. The $u$ quark so formed may unite with an unpaired d quark to produce a ud pair. This process may continue till all the unpaired d quarks are converted into ud pairs. Let a region of space contain thirty-two unpaired $\mathrm{d}$ quarks and sixteen unpaired $\mathrm{u}$ quarks. The sequence of formation of probable nuclei may be described as follows:

$$
\begin{aligned}
& (32 \mathrm{~d}+16 \mathrm{u}) \quad(16 \mathrm{ud}+16 \mathrm{~d}) \\
& \left.\stackrel{e^{-}}{\longrightarrow}(17 \mathrm{ud}+14 \mathrm{~d}), \mathrm{H}-16 \text { (uud }+15 \mathrm{udd}\right) \\
& \left.\stackrel{e^{-}}{\longrightarrow}(18 \mathrm{ud}+12 \mathrm{~d}), \text { He-16 (2uud }+14 \mathrm{udd}\right) \ldots \ldots . \\
& \stackrel{e^{-}}{\longrightarrow}(19 \mathrm{ud}+10 \mathrm{~d}), \text { Li-16 (3uud + 13udd) } \\
& \left.\stackrel{e^{-}}{\longrightarrow}(20 \mathrm{ud}+8 \mathrm{~d}), \text { Be-16 (4uud }+12 \mathrm{udd}\right) \\
& \stackrel{e^{-}}{\longrightarrow}(21 \mathrm{ud}+6 \mathrm{~d}), \mathrm{B}-16 \text { (5uud + 11udd) } \\
& \stackrel{e^{-}}{\longrightarrow}(22 \mathrm{ud}+4 \mathrm{~d}), \mathrm{C}-16 \text { (6uud + 10udd) ....... } \\
& \left.\stackrel{e^{-}}{\longrightarrow}(23 \mathrm{ud}+2 \mathrm{~d}), \mathrm{N}-16 \text { (7uud }+9 \mathrm{udd}\right) \ldots . . . \\
& \stackrel{e^{-}}{\longrightarrow} \text { (24ud), 0-16 (8uud + 8udd) ...... }
\end{aligned}
$$

It is, therefore, evident that the sequence of formation of probable nuclei in regions of more d quarks than $u$ quarks starts with the unstable nuclei of lower atomic numbers and proceeds towards more and more stable nuclei of higher atomic numbers till the most stable nucleus is reached. However, all the nuclei from H-16 through 0-16 are isobars.

The description of formation of nuclei in sections 3 and 4 above through emission of beta particles in stepwise manner (emission of one particle at a time) provides an opportunity for determining the members of a group of nuclei in such a manner that, in going from one member to its adjacent one, the atomic number either increases or decreases by one unit while the mass number remains constant, showing that there is no missing of nuclei between any two adjacent nuclei.

So far, the formation of lighter nuclei was discussed. Now, let us describe the chain of formation of heavier nuclei in a region of space in which there were 330 unpaired u quarks and 384 unpaired d quarks in the early universe. This chain may consist of the following steps.

$$
\begin{aligned}
& (330 u+384 \mathrm{~d}) \rightarrow \mathrm{U}-238(330 \mathrm{ud}+54 \mathrm{~d})(92 \mathrm{uud}+146 \mathrm{udd}) \\
& \stackrel{e^{-}}{\longrightarrow} \mathrm{Np}-238(331 \mathrm{ud}+52 \mathrm{~d})(93 \mathrm{uud}+145 \mathrm{udd}) \\
& \stackrel{e^{-}}{\longrightarrow} \mathrm{Pu}-238(332 \mathrm{ud}+50 \mathrm{~d})(94 \mathrm{uud}+144 \mathrm{udd}) \\
& \left.\stackrel{e^{-}}{\longrightarrow} \text { Am-238 (333ud+ 48d) (95uud + } 143 \mathrm{udd}\right) \\
& \stackrel{e^{-}}{\longrightarrow} \mathrm{Cm}-238(334 \mathrm{ud}+46 \mathrm{~d})(96 \mathrm{uud}+142 \mathrm{udd}) \\
& \left.\stackrel{e^{-}}{\longrightarrow} \text { Bk-238 (335ud+44d) (97uud + } 141 \mathrm{udd}\right) \\
& \stackrel{e^{-}}{\longrightarrow} \mathrm{Cf}-238(336 \mathrm{ud}+42 \mathrm{~d})(98 \mathrm{uud}+140 \mathrm{udd}) \\
& \stackrel{e^{-}}{\longrightarrow} \text { Es-238 (337ud+ 40d) (99uud + } 139 \text { udd) } \\
& \stackrel{e^{-}}{\longrightarrow} \text { Fm-238 (338ud+38d)(100uud + 138udd) }
\end{aligned}
$$




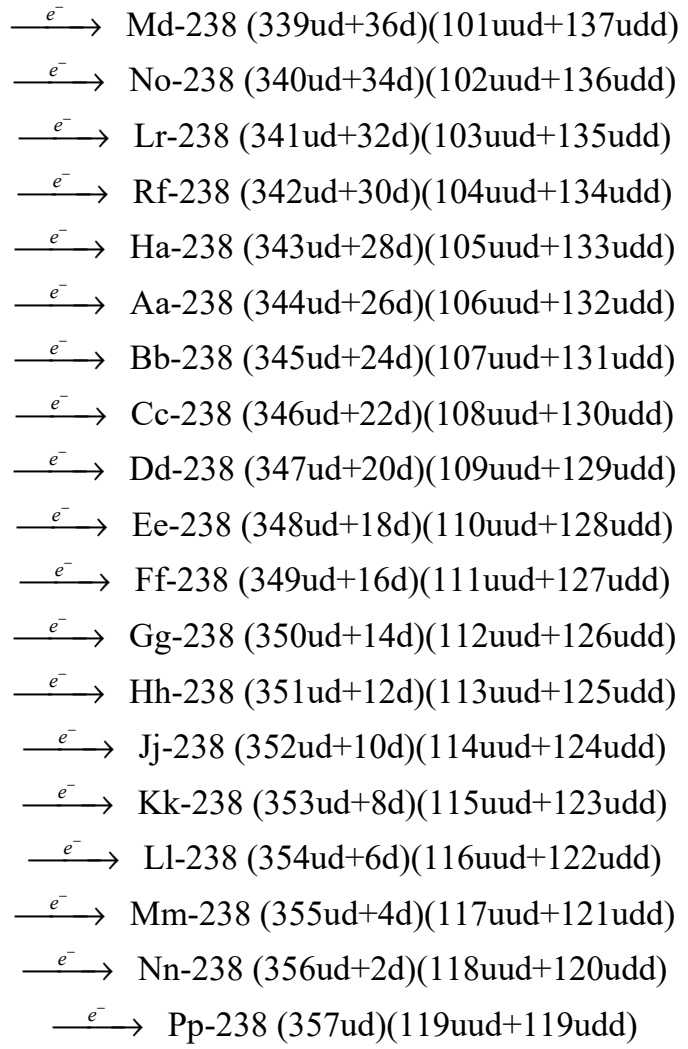

The fourteen nuclei from Aa through Pp are still unknown. However, the Pp-238 nucleus of quark composition (357ud, 119 uud +119 udd) containing no unpaired quarks is expected to be nonradioactive and most stable. All these nuclei form a chain of isobars, showing that the complete transuranium series should contain twenty-eight elements.

\section{Formation of Hydrogen}

As the density of matter constituting the early universe was extremely high, the inter-particles inelastic collisions were likely to be most productive. For example, such collisions between d quarks and ud pairs would be highly probable because both the species are oppositely charged. It was previously deduced (Bhuiyan, 2016) that in the ud pair the u quark is bonded to the d quark through a double bond. Each bond is constituted by an electronposition pair in which the electron is donated from the outermost layer of a d quark and the positron is donated from the outermost layer of a u quark Therefore, the interaction of an isolated d quark with an isolated ud pair may be described as follows.

$$
\begin{gathered}
\text { ud } \rightarrow\left(\mathrm{u}^{\mathrm{e}^{+}-} \mathrm{d}^{\mathrm{e}-}\right) \\
\mathrm{d}^{\mathrm{e}-}+\left(\mathrm{u}^{\mathrm{e}+}-\mathrm{d}^{\mathrm{e}-}\right) \rightarrow \mathrm{du}-\mathrm{d}^{\mathrm{e}-}
\end{gathered}
$$

The terminal $\mathrm{d}^{\mathrm{e}-}$ species may emit an electron and transform into a $u$ quark so that the species dud $\mathrm{d}^{\mathrm{e}-}$ may transform into a proton; thus

$$
\mathrm{du}-\mathrm{d}^{\mathrm{e}-} \rightarrow \mathrm{duu}+\mathrm{e}^{-}
$$

The liberated electron may or may not exist in the surroundings of the proton formed. If it exists in motion around the proton, the combination, duu $+\mathrm{e}-$, would form a hydrogen atom (figure 1).

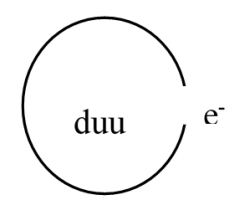

Figure 1. A hydrogen atom 
The Figure 1. shows that one proton may be surrounded by one electron. Two such hydrogen atoms may undergo inelastic collision to produce a hydrogen molecule (Figure 2).

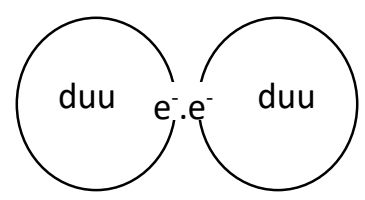

Figure 2. A hydrogen molecule

Therefore, the probability of formation of groups of hydrogen, protons, and electrons was maximum in those regions of space where the population of isolated d quarks and ud pairs was maximum. However, the accumulation of hydrogen in those regions might have led to subsequent formation of stars.

\section{Atomic Structure}

The polymer string (-u-d-) 3 n describes in general the quark composition of nuclei in which u quarks and d quarks are present in the proportion of $1: 1$. The hydrogen atom consists of one proton (uud) which possesses the charge $(+2 / 3+2 / 3-1 / 3)=1$. As the charge of one ud pair is $+2 / 3-1 / 3=+1 / 3$, it takes three ud pairs to make a charge of +1 , suggesting that the quark compostion of the smallest nucleus in the string (-u-d-) $3 n$ should be $3 u d$ (the fractional charge of a nucleus is not acceptable.) In the hydrogen atom the proton is surrounded by one electron. But $3 u d$ represents the quark compostion of one proton and one neutron together. As the charge of a neutron is zero, the composition 3 ud should possess a charge of +1 , implying that a nucleus of composition 3 ud should be surrounded by one electron. So, a nucleus of composion $(3 \times 2)$ ud would be surrounded by two electrons; a nucleus of $(3 \times 3)$ ud, would be surrounded by three electrons; in general, a nucleus of $3 \times n$ ) ud, where $n=1,2,3$, etc. and is also the number of electrons surrounding a nucleus of composition $(3 \times n) u d$, would be surrounded by $n$ electrons. The extranuclear electrons of atoms of some lighter elements are given in Table 1.

Table 1. Extranuclear electrons of some atoms

\begin{tabular}{lll}
\hline Nucleus & Surrounding electron (s) & Atom \\
\hline 3ud & 1 & $\mathrm{D}-2$ \\
6ud & 2 & $\mathrm{He}-4$ \\
9ud & 3 & $\mathrm{Li}-6$ \\
$12 \mathrm{ud}$ & 4 & $\mathrm{Be}-8$ \\
$15 \mathrm{ud}$ & 5 & $\mathrm{~B}-10$ \\
$18 \mathrm{ud}$ & 6 & $\mathrm{C}-12$ \\
$21 \mathrm{ud}$ & 7 & $\mathrm{~N}-14$ \\
$24 \mathrm{ud}$ & 8 & $\mathrm{O}-16$ \\
\hline
\end{tabular}

All the elements represented by the nuclear composition (3Xn)ud, formed simultaneously in space in the early universe, existed as atoms and / or ions in the gaseous state.

\section{More Electrons than Positrons}

In section 5 above, we have discussed the formation of protons and the surrounding electrons through the inelastic collisions between isolated d quark and isolated ud pair in the early universe based on the argument that both the colliding species are oppositely charged. But the chance of a similar collision between an isolated $u$ quark and an isolated ud pair appears to be small in extent because both the species are similarly charged. However, such an interaction, even if it happens, may occur according to the following equations (52) and (53).

$$
\begin{gathered}
\mathrm{u}^{\mathrm{e}+}\left(\mathrm{d}^{\mathrm{e}-}-\mathrm{u}^{\mathrm{e}+}\right) \rightarrow \mathrm{ud}-\mathrm{u}^{\mathrm{e}+} \\
\mathrm{ud}-\mathrm{u}^{\mathrm{e}+} \rightarrow \mathrm{udd}+\mathrm{e}^{+}
\end{gathered}
$$

The final products are neutron and positron. Since the probability of such an interaction is very small, the amounts of neutron and positron formed compared to those of proton and electron would be very small. For this, there would be more protons and electrons than neutrons and positrons in the early universe. Since the expansion just extends this composition to the whole observable universe, the same composition is maintained till the end of the expansion period. Thus, the presence of more electrons than positrons, more protons than neutrons, free atomic 
nuclei, etc., in the socalled "cosmic rays" may support the origin of these species in the early universe. This appears to be supported by the report in Wikipedia on the composition of the primary cosmic rays, which are believed to originate outside of Earth's atmosphere, that these rays are composed of about 99\% atomic nuclei (about $90 \%$ protons, $9 \%$ alpha particles and $1 \%$ nuclei of heavier elements) and about $1 \%$ isolated electrons while positrons/antiprotons form a very small fraction of the total amount.

\section{Nuclear Fission by Neutrons.}

It is now evident that neutrons, protons, the nuclei represented by the polymer $(-\mathrm{u}-\mathrm{d}-)_{3 \mathrm{n}}$ for each value of $\mathrm{n}$ and their related atoms, etc., formed simultaneously in space in the early universe. Since species existed still in close proximity to each other, the probability of collisions between them was very high. Some collisions such as nuclear fission by particles like protons, neutrons, etc., might have started during this period. Due to distortion in the structure, a nucleus may not become as stable as expected. A nucleus of chainlike structure (-u-d-) $3 \mathrm{n}$ may acquire enhanced stability as follows. A nucleus is folded in half. Then one half is placed on the other in such a manner that the $u$ quarks of one half are directly positioned against the $d$ quarks of the other. The positively charged $u$ quarks then interact with the negatively charged d quarks through electrostatic force of attraction. In this way, an ionic bond is formed between the two quarks.

Thus, the covalent bond between the two quarks ( $u$ and $d)$ within the chain $(-u-d-)_{3 n}$ and the interchain ionic bond between them together may make the structure stronger and more stable.

But there exists a certain amount of strain due to distortions at the bend region (B) (Figure 3). The two halves are designated by (A) and (C), respectively. For a symmetrical nucleus, the two halves are equal. In this section we discuss fission of nuclei by neutrons.

$$
\left.\begin{array}{l}
\text { (A) }(u d)_{y}-u-d-u-d-u-d-u-d \\
\text { (C) }(d u)_{z}-d-u-d-u-d-u-d-u
\end{array}\right) \text { B }
$$

Figure 3. A nuclear structure folded in half

If the incident neutron undergoes inelastic collision with the nucleus (Figure 3) at the bend point (B), the latter may undergo splitting into two halves at this point of distortion. Simultaneously, the incident neutron, due to collision with the nucleus, may itself undergo splitting into its constituent parts- one $u$ quark and two $d$ quarks. Since the collision between the nucleus and the neutron occurs at the point B, and if the two segments (A) and (C) are in close proximity to each other, then the $u$ quark of the neutron may attach itself to the terminal $d$ quark of segment (A) while simultaneously the two d quarks of the neutron may attach themselves to the two terminal $u$ quarks of the segment (C). All these are shown in equations (55) and (56) respectively.

$$
\text { A ) } \stackrel{u}{\rightarrow}(\mathrm{ud})_{\mathrm{y}} \quad-\mathrm{u}-\mathrm{d}-\mathrm{u}-\mathrm{d}-\mathrm{u}-\mathrm{d}-\mathrm{u}-\mathrm{d}-\mathrm{u}
$$

(D)

$$
\begin{aligned}
& \stackrel{2 d}{\rightarrow} \quad \text { (C) } \quad(\mathrm{du}) \mathrm{z}-\mathrm{d}-\mathrm{u}-\mathrm{d}-\mathrm{u}-\mathrm{d}-\mathrm{u}-\mathrm{d}-\mathrm{u} \\
& \text { (E) } \quad d \quad d
\end{aligned}
$$

As soon as the segments (D) and (E) return to the ground state, the terminal unpaired u quark in (D) emits a positron and transform into a d quark while simultaneously two neutrons, 2dud, are emitted from the end of the segment $(\mathrm{E})$.

$$
\stackrel{e+}{\rightarrow}(\mathrm{D}) \quad(\mathrm{ud}) \mathrm{y}-\mathrm{u}-\mathrm{d}-\mathrm{u}-\mathrm{d}-\mathrm{u}-\mathrm{d}-\mathrm{u}-\mathrm{d}-\mathrm{d}
$$

$$
(E) \rightarrow \quad(d u)_{z}-d-u-d-u+2 d u d
$$

(G)

Inserting $\mathrm{y}=\mathrm{z}=5$ and then adding $(\mathrm{A})$ and $(\mathrm{C})$ together, one obtains a nucleus of quark composition 18ud which represents C-12 (6uud + 6udd). For the same values of y and z, if one adds (D) and (G) together, then one obtains a nucleus of quark composition ( $16 \mathrm{ud}+\mathrm{u}$ ) which represents $\mathrm{C}-11$ (6uud $+5 \mathrm{udd})$. But if one adds $(\mathrm{F})$ and $\mathrm{G}$ together, then one obtains a nucleus of quark composition $(16 \mathrm{ud}+\mathrm{d})$ which represents B-11 (5uud $+6 \mathrm{udd})$. Therefore, one may write 


$$
\begin{gathered}
\stackrel{e+}{\rightarrow} \mathrm{C}-12+\mathrm{udd} \\
\text { C }-11 \rightarrow \mathrm{C}-11+2 \mathrm{udd}
\end{gathered}
$$

Thus, the collision between $\mathrm{C}-12$ nucleus and a neutron may transform the former first into $\mathrm{C}-11$ nucleus and then into B-11 nucleus with the emission of two neutrons. However the use of fast neutrons may cause the emission of another neutron from the terminal of the segment (F). So, there is the probability of emission of two to three neutrons. In a nutshell, there is probability of emission of $(2+3) / 2=2.5$ neutrons on the average.

This is the case in which the $u$ quark of the incident neutron attaches itself to the half (A) of the nucleus. But there is also the probability for the $u$ quark and two d quarks of the neutron to be attached only to the other half $(\mathrm{C})$ of the nucleus as follows.

$$
\begin{gathered}
\stackrel{u d d}{\longrightarrow}(\mathrm{C}) \quad(\mathrm{ud})_{5}-\mathrm{d}-\mathrm{u}-\mathrm{d}-\mathrm{u}-\mathrm{d}-\mathrm{u}-\mathrm{d}-\mathrm{u} \\
\mathrm{u} \\
\mathrm{d}
\end{gathered}
$$

A high energy neutron may cause the half $(\mathrm{A})$ and the segment $(\mathrm{H})$ to move away from each other and form separate nuclei. The quark composition of $(\mathrm{H})$ is $(7 \mathrm{ud}+\mathrm{u})$ which represents $\mathrm{Li}-5$ nucleus containing one unpaired $\mathrm{u}$ quark. Being unpaired, the $\mathrm{u}$ quark may emit a positron and transform into a $\mathrm{d}$ quark, so that the resulting quark composition is $(7 \mathrm{ud}+\mathrm{d})$ which represents the He-5 nucleus. The He-5 nucleus should be more stable than the Li5 nucleus due to the presence of more stable unpaired $d$ quark in the former. The quark composition of the half (A) is (9ud) which represents the Li-6 nucleus. Thus, a neutron of enough energy may cause the fission of the C-12 nucleus into two nuclei (Li-6 and He-5) of almost equal mass.

These nuclear fission reactions might have started with the appearance of nuclei and neutrons of quark clusters in space in the early universe.

\section{Role of Unpaired Quarks in Nuclei.}

In this section we discuss the role played by unpaired quarks in determining stability (or instability) of a nucleus. One may calculate the number of unpaired quarks ( $u$ and $d$ ) in a nucleus as follows. If $A$ and $Z$ be the mass number and atomic number, respectively, of an atom of an element, then the number of ud pairs in the nucleus is given by

$$
\mathrm{x}=\mathrm{A}+\mathrm{Z}
$$

and the number of unpaired d quarks is given by

$$
\mathrm{y}=\mathrm{A}-2 \mathrm{Z}
$$

The positive $y$ gives the number of unpaired d quarks which exists along with the predicted number, $x$, of ud pairs while the negative $y$ represents the $\mathrm{d}$ quarks that are missing from the predicted number, $\mathrm{x}$, of ud pairs in a nucleus. The following examples may lend support to our arguments.

The predicted number of ud pairs in the $\mathrm{H}-3$ nucleus is $3+1=4$. The value of $y=3-2 \times 1=+1$. So, the quark composition of the H-3 nucleus is $(4 \mathrm{ud}+\mathrm{d})$. If the nuclear chain of $4 \mathrm{ud}$ is folded in half, then each half would consist of two ud pairs. The so folded structure may be represented by either of two figures (Figures 4a and 4b).

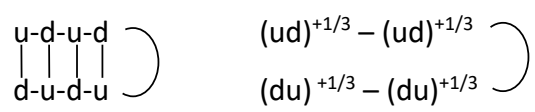

(a)

(b)

Figure 4. Structures of H-3 nucleus when folded in half; (a) interchain u.......d bridging, (b) in units of $(\mathrm{du})^{+1 / 3}$

In structure 4(a) there exists electrostatic attraction interaction between the positively charged u quarks of one half and the negatively charged d quarks of the other half. In structure 4(b) there exists electrostatic repulsive interaction between interchain (ud) ${ }^{+1 / 3}$ units. The actual structure is a hybrid of both the structures; that is, both the structures would exist in reversible equilibrium with each other. 
The negatively charged unpaired d quark may remain attached to any one of the (ud) ${ }^{+1 / 3}$ and $(\mathrm{du})^{+1 / 3}$ units in structure (4b); that is, it may delocalize throughout the space between the two folded halves but it may encounter repulsive interaction with the negatively charged d quarks situated in both the halves in structure (4a). This repulsive interaction may make the unpaired d quark somewhat unstable, for which it may emit an electron and transform into a u quark. The resulting nucleus may possess the quark composition given by the following equation

$$
4 \mathrm{ud}+\mathrm{d} \stackrel{e-}{\rightarrow} 4 \mathrm{ud}+\mathrm{u}
$$

But the positively charged u quark so produced may also find it difficult to delocalize through the space between the two folded halves. This will make the $\mathrm{u}$ quark unstable, for which it may emit one positron and transform into a d quark. The quark composition of the resulting nucleus is given by

$$
4 \mathrm{ud}+\mathrm{u} \stackrel{e+}{\rightarrow} \quad 4 \mathrm{ud}+\mathrm{d}
$$

It is evident that the nuclei of quark compositions $(4 u d+d)$ and $(4 u d+u)$ may exist in reversible equilibrium with each other in the same region of space. This means that both the nuclei will exist together in the same region of space. The quark composition $(4 u d+d)$ represents the H-3 nucleus (uud $+2 u d d)$ while the one $(4 u d+u)$ may represent the He-3 nucleus ( 2 uud+udd). It, therefore, follows that both the nuclei would remain in association in the same region of space. It is found that H-3 and He-3 exist in association in the upper atmosphere (Cotton \& Wilkinson, 1972), suggesting that both the nuclei were formed in space in the early universe simultaneously with the other nuclei. Probably nuclei, atoms, and molecules still keep floating in space throughout the whole universe.

\section{Formation in Space of Precursors of Living Organisms.}

The amino acids, nitrogenous bases, phosphoric acid, sugars, etc., form the precursors of DNAs and RNAs which, in turn, form the genetic materials of all living organisms as well as of viruses. Scientists believe that comets or asteroids carried the precursors of amino acids and nitrogenous bases in meteorites. Recently (Meinert et al., 2016; CNRS, 2016), the astrophysicists produced dust grains coated with ice, the raw material of comets, by placing a mixture of water, methanol and ammonia in a high vacuum chamber at $200^{\circ} \mathrm{C}$ and then irradiating the mixture with UV. After irradiation, the mixture was warmed to room temperature. On analysis, the sample was found to contain several sugars including ribose. Although the results form a key step in understanding the origin of RNA, yet the sugar ribose has not yet been found in real comets.

Formation of sugars including ribose and other precursors of DNA and RNA in space in the early universe may be elucidated as follows. Formation of hydrogen through interaction of d quarks with ud pairs is described in section 5 above. Generation of other elements including carbon, nitrogen, oxygen, etc., by the polymer $(-\mathrm{u}-\mathrm{d}-)_{3 \mathrm{n}}$ is shown in Table-1. The temperature of the early universe was very high, for which hydrogen and all the elements represented by (-u-d-) $)_{3 n}$ were supposed to exist as atoms/ ions in the gaseous state. As the temperature started to fall, the atoms started to form molecules and compounds. Hydrogen formed hydrogen (gas) molecule; carbon and oxygen formed carbon monoxide; hydrogen and oxygen formed water; nitrogen and hydrogen formed ammonia; carbon monoxide and hydrogen formed methanol. Thus, water, methanol, and ammonia, which are precursors of ribose, could be formed in space in the early universe. Nucleotides, which form the building blocks (units) in DNA and RNA, consist of three components - a phosphate group, a ribose, and purine or pyrimidine as a heterocyclic base. Formation of ribose is already described above. Phosphorus was oxidized by oxygen to various oxidespentoxide, trioxide, etc., which combined with water to produce phosphoric acids of various compositions. Purines and pyrimidines were formed from compounds of lower molecular weights. For example, aspartic acid, carbon dioxide, glutamine, glycine, and formal tetrahydrofolate, formed earlier, combined together to form the nucleus of purine. As cooling continued, all the three components- phosphorus acid, ribose, and the heterocyclic base combined together to produce one block (or unit) in the DNA/ RNA threads. All these products existed in the gaseous state. But, on considerable cooling, the gaseous water first transformed into fog and then into cloud with precursors embedded in it. The sufficiently cooled cloud produced rain water which carried the precursors along with other substances on the surface of a planet like the earth where they combined to produce DNAs, RNAs, proteins, etc., of plants and animals. Alternatively, the rain water might have, due to supercooling, transformed into ice in which the precursors were kept frozen for a long long time before the ice melted into water again and the simultaneous evolution of all kinds of living organisms including plants and animals started. But some of all precursors were left free in rocks, soil, water and the atmospheric air. The smaller precursors in these locations may still continue to form larger precursors, such as nucleotides which are the building blocks of DNA, RNA, etc. After passing into the systems of existing plants, animals, bacteria, germs, viruses, etc., via food, water and air, these nucleotides may join at the end points of the DNAs in them, causing the DNAs to increase in length. The lengthened DNAs containing more bases may exhibit modified characteristics, for which the bacteria, germs and viruses may start growing more and more resistant to the currently used drugs and medicines. Pests may start to 
develop more resistance to pesticides while herbs may become resistant to herbicides. Further, the precursors in the atmospheric air may produce new form of bacteria, germs and viruses while those in the soil on earth may produce new form of pests and herbs. The new pests may develop resistance to the currently employed pesticides while the new herbs may develop resistance to the currently used herbicides. The new viruses, germs and bacteria may develop resistance to antibiotics. The human beings, who live on foods supplied via modified and new plants, animals, creatures, etc., may continue to become modified in behavior, intelligence and other activities. This shows that this process of natural evolution in plants, animals (including human beings) and other living organisms (including bacteria, germs and viruses) may continue till the end of the period of expansion of the universe.

If investigation of rocks and stones, sand, soil and water of earth's surface as well as earth's atmosphere reveals the presence of these precursors or their constituents in them, then the formation of these precursors in space in the early universe will be substantiated. However, the first human child might have looked around and asked the question: who (and where) am I? Probably every human child asks the same question till today through his / her cries just after birth.

\section{Conclusions}

a) Immediately after the formation of $u$ quarks and d quarks from electron- positron pairs, the nuclei of different elements started to form in space in the early universe simultaneously through different pathways. Some of these pathways are described. Each pathway leads to the formation of the most stable nucleus through transformation of its less stable isobars. Such stable nucleus belongs to the polymer (-u-d-) $)_{3 \mathrm{n}}$. For example, the stable nucleus N-14 was formed through stepwise transformation of the less stable isobars Al-14, Mg-14, Na-14, Ne-14, F-14 and 014 in that order in a region of space where there were more $u$ quarks than $d$ quarks while the stable $0-16$ nucleus was formed through stepwise transformation of the less stable isobars H-16, He-16, Li-16, Be-16, B-16, C-16 and $\mathrm{N}-16$ in that order in a region of space where there were more d quarks than u quarks.

b) The transformation of less stable isobars into more stable ones in a region was accompanied by emission of electrons/positrons. So, if these regions were contiguous to each other, then mutual annihilation of these electrons and positrons produced radiations, suggesting that the early universe was dominated by radiation.

c) The formation of the most stable nucleus of atomic number 119 could occur through 26 radioactive nuclei starting from U-238. All these nuclei including U-238 form isobars of the nucleus of atomic number 119.

d) Hydrogen produced through interaction of isolated d quarks with isolated ud pairs might form fuels for stars. For example, our sun uses hydrogen as fuel and burns it to produce helium, heat and light.

e) Nuclear fission reactions might have started with the simultaneous appearance of neutrons, protons, etc, in space in the early universe. Our sun is believed to produce helium through fusion of hydrogen. As an extremely high temperature is needed to cause fusion of nuclei, one my derive such high temperature from the fission of heavier nuclei. In hydrogen bomb this high temperature is obtained for the fusion of hydrogen by the fission of a heavier nucleus such as uranium. Our sun, formed some five thousand million years ago, contains about 2 percent of heavier nuclei. It may, therefore, be presumed that neutrons, protons, etc., present in the sun may cause fission of these nuclei to produce the high temperature needed to cause the fusion of hydrogen, suggesting that the fission reactions had started in the early universe.

f) Existence of H-3 and He-3 nuclei in reversible equilibrium in upper atmosphere may provide evidence of formation in space of these and other nuclei simultaneously from $u$ quarks and d quarks in the early universe.

g) As all elements including hydrogen, carbon, etc., formed simultaneously, the processes such as oxidation, reduction, ring formation, cyclization, etc., led to the formation of compounds of lower molecular weights which later formed the precursors of DNAs and RNAs. These precursors were carried by rain water to the surface of a cooled planet where they combined to form DNAs and RNSs of all living organisms.

h) The process of natural evolution of living organisms may continue till the end of the period of expansion of the universe.

\section{References.}

Bhuiyan, A. L. (2010). The Nature of the Exchange Forces. Physics Essays, 23(1), 97-103. http://dx.doi.org/10. $4006 / 1.3297939$

Bhuiyan, A. L. (2013). Qualitative Nuclear Structures in Terms of Clusters of Quarks. Physics Essays, 26(2), 223236. http://dx.doi.org/10.4006/0836-1398-26.2.223

Bhuiyan, A. L. (2015). Quantum Description of Gravity. Physics Essays, 28(2), 164-166. http://dx.doi.org/10/ 4006/0836-1398-28.2.164 
Bhuiyan, A. L. (2016). Application of the Concept of Nuclear ud pair Clusters. Applied Physics Research, 8(2), 29-37. http://dx.doi.org/10.5539 /apr.v8n2p29

Science Daily. (2016). CNRS: Origin of Life: An artificial comet holds the missing piece.

Cotton, F. A., \& Wilkinson, G. (1972). Advanced Inorganic Chemistry: A Comprehensive Text (3rd ed., p. 149). New York: Interscience.

Hawking, S. W. (1988). A Brief History of Time: From the Big Bang to Black Holes (Bantam edition reprinted 1996, pp. 139-141). New York: Bantam Books.

Meinert, C., Myrgorodska, I., De Marcellus, P., Buhse, T., Nahon, L., Hoffmann, S. V., ... \& Meierhenrich, U. J. (2016). Ribose and related sugars from ultraviolet irradiation of interstellar ice analogs. Science, 352(6282), 208-212. http:/dx.doi.org/10.1126/science. aad8136

Seking, M. (2012). The Cantor Set constructed from an infinite Number of Quarks Constituting the Nucleon and the Dark Matter. Applied Physics Research, 4(4), 68-72. http:/dx.doi.org/10.5539/apr.v4n4p68

\section{Copyrights}

Copyright for this article is retained by the author(s), with first publication rights granted to the journal.

This is an open-access article distributed under the terms and conditions of the Creative Commons Attribution license (http://creativecommons.org/licenses/by/4.0/). 